\title{
Discovering the pathogenesis of autoimmune diseases at the 9th International Congress of Autoimmunity, Nice, France, 2014
}

\author{
Carlo Perricone $\cdot$ Guido Valesini
}

Published online: 3 December 2014

(C) Springer Science+Business Media New York 2014

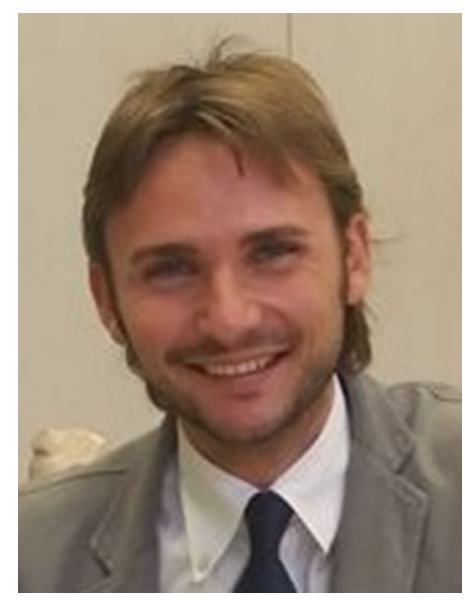

Carlo Perricone

It was probably a sign that the 9th International Congress on Autoimmunity was held in the astonishing setting of the Acropolis, Congress venue, in Nice. The word Acropolis comes from Greek from akros, "topmost," and polis, "city": It was indeed topmost the level of the contributions from the over 2000 scientists of the polis of the Autoimmunity Congress. The huge number of participants led to pulsating discussions on different aspects of autoimmunity. In this issue of Immunologic Research, are gathered papers referring to the novel findings on pathogenesis beginning from the genetic background. Reeves et al. [1] reviewed the role of the endoplasmic reticulum aminopeptidase 1 (ERAP1) in ankylosing spondylitis (AS). This zinc metallopeptidase has established roles in biological processes such as antigen processing for major histocompatibility complex (MHC) class I presentation, cytokine receptor shedding, angiogenesis/blood pressure regulation and activation of macrophage [2]. ERAP1 is interesting for its role in MHC I antigen-processing pathway where the generation of optimal antigens for loading onto MHC I molecules are essential for the stable expression of MHC I at the cell surface to circulating $\mathrm{CD}^{+} \mathrm{T}$ cells. The ERAP1 processing can foresee either the "molecular ruler" mechanism, in which ERAP1 trims the peptide precursor to the correct length for binding with the molecule itself acting as a length template, or a mechanism in which the MHC I acts as the template required by ERAP1. Nonetheless, ERAP1 is interesting also because in a way it can differentiate the pathogenesis of different autoimmune conditions. The

C. Perricone $(\bowtie) \cdot$ G. Valesini

Reumatologia, Dipartimento di Medicina Interna e Specialità Mediche, Sapienza Università di Roma, Viale del Policlinico 155, 00161 Rome, Italy

e-mail: carlo.perricone@gmail.com genome-wide analysis studies have indeed found an association with a number of disorders sharing clinical and pathogenic features with AS: Behçet's disease, psoriasis and psoriatic arthritis (PsA) [3]. These have in common uveitis, a (relatively) frequent axial involvement, and the interleukin IL-17/IL-23 pathway "signature." Thus, it could be hypothesized that the combination of the HLA locus (B27 for AS and PsA, B51 for Behçet) plus the presence of other genetic variants, including ERAP1, may be responsible for different clinical onset. It is not surprising that we failed to find any association with systemic lupus erythematosus (SLE) [4]. In this disease, the role of IL-17 is better characterized, while that of IL-23 is less clear. Indeed, there is no association with IL-23 genetic variants and SLE [5]. This evidence may suggest that ERAP1 is quite associated with an IL-23 rather than an IL-17 milieu.

Moving forward to the identification of clinical entities, the new star in the firmament of autoimmunity is the "Autoimmune (Auto-inflammatory) Syndrome induced by Adjuvants" (ASIA) [6]. Agmon-Levin et al. [7] aimed at assessing the association between fibromyalgia or chronic fatigue and hepatitis B vaccination (HBVv). They found that all patients fulfilled the criteria for ASIA syndrome, suggesting that chronic fatigue syndrome and fibromyalgia can be part of this disorder, at least transiently. Interestingly, there was a high prevalence of patients testing positive to autoantibodies (71\%). In a previous study, Zafrir et al. described 93 cases of ASIA following HBVv. The mean age of onset, the great prevalence of neurological disturbances and general symptoms suggest an overlap between chronic fatigue syndrome and fibromyalgia in ASIA. Indeed, Zafrir et al. [8] already could identify central pain syndrome in $20.5 \%$ of the cases, suggesting that HBVv may be associated in some cases with the onset of chronic fatigue syndrome and fibromyalgia. It must be 
underlined that chronic fatigue syndrome and fibromyalgia are often associated with autoimmune disorders and represent a burden for a relatively high percentage of patients with Sjögren's syndrome [9]. Nonetheless, Sjögren's syndrome has been itself collected under the umbrella of the ASIA, suggesting a further link between these disorders and the central pain syndrome [10].

Despite all these achievements in the field, the diagnosis of ASIA is still puzzling. Esposito et al. [11] have analyzed the data on the possible development of the syndrome in pediatric patients. Several aspects need a comment. First, the literature on the matter is scarce, possibly because the onset of an autoimmune response after adjuvants' exposure often requires time [12]. Secondarily, children are less exposed to specific adjuvants, such as silicone, while sick building syndrome has been described also in the pediatric background. Consequently, few post-vaccine phenomena can be categorized being ASIA syndrome, possibly associated with the development of protean clinical pictures ranging from Guillain-Barrè syndrome (GBS) to narcolepsy [13]. However, additional studies are needed to clarify such post-adjuvant onset of autoimmunity in the pediatric age, while the benefits of vaccines should never be disregarded.

Antonelli et al. [14] have brilliantly addressed the role of cytokines in $\mathrm{HCV}$-autoimmune-related disorders. These are still a matter for our society; moreover, a better knowledge of these diseases may unveil key mechanisms shared by other autoimmune conditions. Cytokines work as intercellular mediators in both viral control and liver damage. The crucial step in disease progression and phenotype is the disruption in cytokines balance. Thus, the genetic background of each individual mixed with the HCV infection leads to the different clinical pictures. IFN- $\gamma$-inducible CXCL9, -10 and -11 chemokines seem the responsible for inflammatory infiltrate recruitment into the liver parenchyma owing to the inability to control the infection process, which result in sustained liver damage and eventually in liver cirrhosis. Moreover, new actors are now in the scene, such as IL-28B polymorphisms and circulating CXCL10, which may represent prognostic markers for $\mathrm{HCV}$ treatment efficacy in HCV genotype-1 infection. Finally, the usefulness of rituximab (anti-CD20) in mixed cryoglobulinemia underlines the importance of B cell-derived cytokines in disease pathogenesis and progression [15].

Besides cytokines, hormones play a major role in autoimmunity. Clinical and experimental data clearly indicate that females have enhanced humoral and cellular immune responses compared with males [16]. Furthermore, it is largely described the higher prevalence of females compared with males during the childbearing ages among patients with systemic lupus erythematosus (SLE), primary biliary cirrhosis, autoimmune thyroid diseases and systemic sclerosis.
On the other side, few autoimmune disorders display an almost equal or inverse ratio, such as rheumatoid arthritis (RA) and psoriatic arthritis (PsA). Dar et al. [17] found that there is no difference in the sex of offspring form patients with SLE, RA or PsA. There are several conclusions to this evidence. The most intuitive is that the determination of sex in newborns, besides being possibly influenced by environmental factors, is less dependent on hormonal factors. Indeed, it was previously observed a male predominance of $69 \%$ among offspring of women with SLE compared to $51.2 \%$ among the offspring of women with no connectivetissue disorders [18]. It is more likely that other unrecognized factors contributed to that observation.

Another intriguing argument links autoimmunity with cancer [19]. It has been showed that autoimmune phenomena may develop after neoplasia. Maroun et al. [20] added another dowel to this mosaic demonstrating that autoantibodies to important centrosome antigens can be associated with breast cancer. The anticentrosome antibodies are often prodromal of the development of systemic sclerosis especially when Raynaud's phenomenon is present. On the other hand, the same autoantibodies may be present early during carcinogenesis, suggesting their usefulness as biomarkers of early breast cancer. The existence of a higher rate of $\mathrm{BC}$ in patients with systemic sclerosis may now have found an explanation [21].

Nonetheless, the utility of specific autoantibodies in disease diagnosis is clearer and clearer. In the last years, the assessment of the antibodies directed toward citrullinated proteins/peptides (ACPA) revolutionized the management of RA. Watad et al. [22] found that ACPA had with a positive predictive value of $43 \%$ and a negative predictive value of $95 \%$, and only $20 \%$ of ACPA tests referred to a tertiary center were found to be positive. ACPA should then be used as an exclusion criterion in the process of evaluating a patient with rheumatic disease. ACPA value in diagnosis of RA remains undebatable; however, better standardization of the assays and a more stringent prescription in routine practice are the unmet needs [23].

Switching from bench to bedside, there is the feeling of the need of more effective, safer and cheaper drugs. Moreover, an improved administration of known immunosuppressants is desirable in the management of autoimmune diseases [24]. Romão et al. [25] performed an extensive review evaluating the factors predicting methotrexate toxicity. A number of pharmacogenetic studies have been completed, including SLC19A, ABCB1, ABCC2, ABCG2, FPGS, GGH, DHFR, MTHFD1, MTHFR, MTR, MTRR, SHMT1, TYMS, ATIC, ADORA2A and AMPD1. Despite such extensive studies, pharmacogenetic data have not been consistent enough in order to be clinically useful, and further studies are strongly demanded. 
Selmi et al. [26] discussed what is cooking in the pot of treatment for autoimmune diseases. From one side, small molecules will represent a step aside from "classic" biologics since they have been developed to target intracellular signaling. Thus, not only the mechanism of these molecules is changed, but also we will deal with different administration routes and, unfortunately, different adverse events that by now seem to limit their usage. Moreover, the number of biologics is rapidly growing, with an expanding set of targeted pathogenic pathways. However, the relatively low rate of success of ongoing trials should also bring into question whether the way such trials are designed has been correct so far. Indeed, the very high costs and the urgent need for new treatments are a burden for this society that cannot waste money while patients simply cannot wait [27].

An everlasting player in the treatment of autoimmune diseases remains intravenous immunoglobulin (IVIg). IVIg is largely used as replacement therapy in patients with antibody deficiencies. Nonetheless, due to their beneficial effects including Fc receptor blockade, inhibition of cytokines and growth factors, modulation of macrophages and dendritic cells, enhancement of regulatory $\mathrm{T}$ cells, and modulation of B cells through the Fc $\gamma$ RIIb receptor and CD22, they have been proved useful in the treatment of autoimmune diseases. Mitrevski et al. [28] have depicted a new way by which IVIg therapy may drive the $\mathrm{B}$ cells of patients with common variable immunodeficiency (CVID) to down-regulate CD21 expression and to assume the peculiar phenotype of the anergic-like, apoptosis prone $\mathrm{CD} 21^{\text {low }} \mathrm{B}$ cells. These are spontaneously expanded in a subset of CVID and in some other immunologic disorders. More than that, such CD $21^{\text {low }}$ B cells newly generated after IVIg infusion undergo spontaneous apoptosis upon in vitro culture. The possible meaning is that IVIg therapy may constrain antibody responses by inducing $\mathrm{B}$ cell depletion through differentiation into $\mathrm{CD} 21^{\text {low }} \mathrm{B}$ cells that undergo accelerated apoptosis. Indeed, also previous studies suggest that IVIg therapy may induce in human B cells functional and phenotypic changes similar to anergy [29]. Taken together, these data suggest that IVIg may find their efficacy in B cell driven autoimmune diseases, as it was observed in refractory cases of SLE [30]. A better understanding of the exact connection between $B$ cells and IVIg may allow the development of tailored therapy for patients with autoimmune diseases.

Notwithstanding, Maddur et al. [31] added another bullet at the IVIg weapon. They found that the beneficial effects of IVIg treatment in Guillain-Barré syndrome might be dependent on its capability of lowering the number of Th1 and Th17 cells with a concomitant expansion of $\mathrm{T}_{\text {reg }}$ cells in GBS patients. This is in line with the observation of an overall preserved number of $\mathrm{T}$ cells after IVIg administration [32]. The $\mathrm{T}_{\text {reg }}$ cells exhibited have an increased $\mathrm{T}$ cell suppressive function, which in turn may reduce the immune response in course of the disease [33].

To conclude, in a few years, the markers that now we use for diagnosis, such as the genes, may become targets of an effective therapy in a new dawn for autoimmunity. Little by little, the pathophysiology of autoimmune diseases is being revealed, and this Congress helped us in expanding our knowledge. It is only by sharing of ideas and transverse application of sightings that the medicine of autoimmunity can improve.

\section{References}

1. Reeves E, Elliott T, James E, Christopher J, Edwards CJ. ERAP1 in the pathogenesis of ankylosing spondylitis. Immunol Res. 2014. doi:10.1007/s12026-014-8576-2.

2. Fierabracci A, Milillo A, Locatelli F, Fruci D. The putative role of endoplasmic reticulum aminopeptidases in autoimmunity: insights from genomic-wide association studies. Autoimmun Rev. 2012;12(2):281-8.

3. Genetic Analysis of Psoriasis Consortium, the Wellcome Trust Case Control Consortium 2, Strange A, et al. A genome-wide association study identifies new psoriasis susceptibility loci and an interaction between HLA-C and ERAP1. Nat Genet. 2010;42(11):985-90. doi:10.1038/ng.694.

4. Ciccacci C, Perricone C, Ceccarelli F, Rufini S, Di Fusco D, Alessandri C, Spinelli FR, Cipriano E, Novelli G, Valesini G, Borgiani P, Conti F. A multilocus genetic study in a cohort of Italian SLE patients confirms the association with STAT4 gene and describes a new association with HCP5 gene. PLoS ONE. 2014;9(11):e111991.

5. Sánchez E, Rueda B, Callejas JL, Sabio JM, Ortego-Centeno N, Jimenez-Alonso J, López-Nevot MA, Martín J. Analysis of interleukin-23 receptor (IL23R) gene polymorphisms in systemic lupus erythematosus. Tissue Antigens. 2007;70(3):233-7.

6. Perricone C, Colafrancesco S, Mazor RD, Soriano A, AgmonLevin N, Shoenfeld Y. Autoimmune/inflammatory syndrome induced by adjuvants (ASIA) 2013: unveiling the pathogenic, clinical and diagnostic aspects. J Autoimmun. 2013;47:1-16.

7. Agmon-Levin N, Zafrir Y, Kivity S, Balofsky A, Amital H, Shoenfeld Y. Chronic fatigue syndrome and fibromyalgia following immunization with the hepatitis B vaccine-another angle of the "Autoimmune (Auto-inflammatory) Syndrome induced by Adjuvants' (ASIA). Immunol Res. 2014. doi:10.1007/s12026014-8604-2.

8. Zafrir Y, Agmon-Levin N, Paz Z, Shilton T, Shoenfeld Y. Autoimmunity following hepatitis $\mathrm{B}$ vaccine as part of the spectrum of 'Autoimmune (Auto-inflammatory) Syndrome induced by Adjuvants' (ASIA): analysis of 93 cases. Lupus. 2012;21(2):146-52.

9. Iannuccelli C, Spinelli FR, Guzzo MP, Priori R, Conti F, Ceccarelli F, Pietropaolo M, Olivieri M, Minniti A, Alessandri C, Gattamelata A, Valesini G, Di Franco M. Fatigue and widespread pain in systemic lupus erythematosus and Sjögren's syndrome: symptoms of the inflammatory disease or associated fibromyalgia? Clin Exp Rheumatol. 2012;30(6 Suppl 74):117-21.

10. Colafrancesco S, Perricone C, Priori R, Valesini G, Shoenfeld Y. Sjögren's syndrome: another facet of the autoimmune/inflammatory syndrome induced by adjuvants (ASIA). J Autoimmun. 2014;51:10-6. 
11. Esposito S, Prada E, Mastrolia MV, Tarantino G, Codecà C, Rigante D. Autoimmune/inflammatory syndrome induced by adjuvants (ASIA): clues and pitfalls in the pediatric background. Immunol Res. 2014. doi:10.1007/s12026-014-8586-0.

12. Agmon-Levin N, Hughes GRV, Shoenfeld Y. The spectrum of ASIA: 'Autoimmune (Auto-inflammatory) Syndrome induced by Adjuvants'. Lupus. 2012;21:118-20.

13. Perricone C, Agmon-Levin N, Shoenfeld Y. Novel pebbles in the mosaic of autoimmunity. BMC Med. 2013;4(11):101.

14. Antonelli A, Ferrari SM, Ruffilli I, Fallahi P. Cytokines and HCV-related autoimmune disorders. Immunol Res. 2014. doi:10. 1007/s12026-014-8569-1.

15. Visentini M, Conti V, Cristofoletti C, Lazzeri C, Marrapodi R, Russo G, Casato M, Fiorilli M. Clonal expansion and functional exhaustion of monoclonal marginal zone B cells in mixed cryoglobulinemia: the yin and yang of HCV-driven lymphoproliferation and autoimmunity. Autoimmun Rev. 2013;12(3):430-5.

16. Shoenfeld Y, Tincani A, Gershwin ME. Sex gender and autoimmunity. J Autoimmun. 2012;38(2-3):J71-3.

17. Dar L, Shalev V, Weitzman D, Chodick G, Arnson Y, Amital H. No male predominance in offspring of women with rheumatoid arthritis or systemic lupus erythematosus. Immunol Res. 2014. doi:10.1007/s12026-014-8603-3.

18. Novack V, Erez O, Novack L, Jotkowitz A, Meir A, Mazor M. Sex distribution of newborns to mothers with systemic lupus erythematosus. Epidemiology. 2006;17(3):341-2.

19. Vermeersch P, Bossuyt X. Prevalence and clinical significance of rare antinuclear antibody patterns. Autoimmun Rev. 2013;12(10): 998-1003.

20. Maroun M-C, Olivero O, Lipovich L, et al. Anti-centrosome antibodies in breast cancer are the expression of autoimmunity. Immunol Res. 2014. doi:10.1007/s12026-014-8582-4.

21. Colaci M, Giuggioli D, Vacchi C, et al. Breast cancer in systemic sclerosis: results of a cross-linkage of an Italian rheumatologic center and a population-based cancer registry and review of the literature. Autoimmun Rev. 2014;13:132-7.

22. Watad A, Agmon-Levin N, Gilburd B, Lidar M, Amital H, Shoenfeld Y. Predictive value of anti-citrullinated peptide antibodies-a real life experience. Immunol Res. 2014. doi:10.1007/ s12026-014-8566-4.

23. Alessandri C, Priori R, Modesti M, Mancini R, Valesini G. The role of anti-cyclic cytrullinate antibodies testing in rheumatoid arthritis. Clin Rev Allergy Immunol. 2008;34(1):45-9.
24. Conti F, Perricone C, Ceccarelli F, Valesini G. Rituximab treatment of systemic lupus erythematosus in controlled trials and in clinical practice: two sides of the same coin. Autoimmun Rev. 2010;9(11):716-20.

25. Romão VC, Lima A, Bernardes M, Canhão H, Fonseca JE. Three decades of low-dose methotrexate in rheumatoid arthritis: Can we predict toxicity? Immunol Res. 2014. doi:10.1007/s12026-0148564-6.

26. Selmi C, Generali E, Massarotti M, Bianchi G, Sciré CA. New treatments for inflammatory rheumatic disease. Immunol Res. 2014. doi:10.1007/s12026-014-8565-5.

27. Ebrahim S, Sohani ZN, Montoya L, Agarwal A, Thorlund K, Mills EJ, Ioannidis JP. Reanalyses of randomized clinical trial data. JAMA. 2014;312(10):1024-32.

28. Mitrevski M, Marrapodi R, Camponeschi A, Lazzeri C, Todi L, Quinti I, Fiorilli M, Visentini M. Intravenous immunoglobulin replacement therapy in common variable immunodeficiency induces B cell depletion through differentiation into apoptosisprone CD21low B cells. Immunol Res. 2014. doi:10.1007/ s12026-014-8599-8.

29. Séité JF, Goutsmedt C, Youinou P, Pers JO, Hillion S. Intravenous immunoglobulin induces a functional silencing program similar to anergy in human B cells. J Allergy Clin Immunol. 2014;133:181-8.

30. Perricone R, De Carolis C, Kröegler B, Greco E, Giacomelli R, Cipriani P, Fontana L, Perricone C. Intravenous immunoglobulin therapy in pregnant patients affected with systemic lupus erythematosus and recurrent spontaneous abortion. Rheumatology (Oxford). 2008;47(5):646-51.

31. Maddur MS, Rabin M, Hegde P, Bolgert F, Guy M, Vallat JM, Magy L, Bayry J, Kaveri SV. Intravenous immunoglobulin exerts reciprocal regulation of Th1/Th17 cells and regulatory $\mathrm{T}$ cells in Guillain-Barré syndrome patients. Immunol Res. 2014. doi:10. 1007/s12026-014-8580-6.

32. Hou HQ, Miao J, Feng XD, Han M, Song XJ, Guo L. Changes in lymphocyte subsets in patients with Guillain-Barré syndrome treated with immunoglobulin. BMC Neurol. 2014;14(1): 202.

33. Kieseier BC, Lehmann HC, Hörste GMZ. Autoimmune diseases of the peripheral nervous system. Autoimmun Rev. 2012;11(3): $191-5$. 